\title{
Sobre las estrategias discursivas, hipertextuales y multimediales orientadas a la creación de espacios de aprendizaje en entornos online
}

\author{
Multimedia, hypertextual and discursive strategies aimed to develop online learning spaces
}

\author{
Sobre as estratégias discursivas, hipertextuais e multimidiáticas voltadas à criação de \\ espaços de aprendizagem em ambientes on-line
}

\begin{abstract}
Guadalupe Álvarez
Centro de Investigaciones en Antropología Filosófica y Cultural, Consejo Nacional de Investigaciones Científicas y Técnica (CIAFIC-CONICET), Instituto del Desarrollo Humano, Universidad Nacional de General Sarmiento (IDH-UNGS), Buenos Aires, Argentina, 54-11-47760913, galvarez@ungs.edu.ar
\end{abstract}

\begin{abstract}
RESUMEN
Con los avances de las Tecnologías de la Información y la Comunicación y el continuo desarrollo de dinámicas interactivas multimediales en sitios webs y plataformas de e-learning, la relación entre aprendizaje y discurso adquiere una nueva y singular vigencia. El objetivo del presente artículo es analizar dicha relación considerando la conceptualización de aprendizaje ofrecida por Marton et al. (2004) y los principios fundamentales de las semiótica social multimedial. Desde esta perspectiva, se estudian las estrategias (Lemke, 1997) discursivas, hipertextuales y multimediales utilizadas por docentes, tutores y alumnos para configurar los llamados espacios de aprendizaje en las diferentes materias de un Máster interuniversitario en formación de profesorado de calidad. Este análisis muestra que las estrategias hipertextuales y multimediales son infrecuentes y solo empleadas por los tutores. Por otra parte, tanto los tutores como los alumnos utilizan estrategias que apuntan, fundamentalmente, al "aprendizaje en un sentido restringido".
\end{abstract}

Palabras clave: educación online, aprendizaje, discurso, multimedia.

\begin{abstract}
With the Information and Communication Technology, and the continuous development of interactive multimedia dynamics in web sites and e-learning platforms, the relationship between learning and discourse has come into a new place. This paper aims to analyze this relationship in online contexts considering Marton et al. (2004) learning concept, and the basic guidelines of multimodal social semiotic. From this perspective, multimedia, hypertextual and discursive strategies aimed to develop online learning spaces are analyzed in the different subjects of an Interuniversity's Master of quality faculty formation. The analysis shows that hypertextual and multimedia strategies are infrequent, and only used by tutors. Furthermore, both tutors and students strategies are directed to a "limited learning" development.
\end{abstract}

Key words: online education, learning, discourse, multimedia.

\section{RESUMO}

Com os avanços das Tecnologias da Informação e a Comunicação e com o contínuo desenvolvimento de dinâmicas multimidiáticas interativas em sites e plataformas de e-learning, a relação entre aprendizagem e discurso adquire nova e singular vigência. Tendo em vista o conceito de aprendizagem de Marton et al. (2004) e os princípios fundamentais da semiótica social multimídia, analisa-se essa relação. A partir de essa perspectiva, estudam-se as estratégias (Lemke, 1997) discursivas, hipertextuais e multimidiáticas utilizadas por docentes, orientadores e orientandos a fim de configurar os chamados espaços de aprendizagem em diferentes disciplinas de um Mestrado interuniversitário voltado para a formação de professores de qualidade. A análise mostra que as estratégias hipertextuais 
e multimidiáticas são infrequentes e empregadas apenas pelos orientadores. Por outro lado, tanto para orientadores como orientandos, as estratégias se destinam, fundamentalmente, à "aprendizagem em sentido restrito".

Palavras chave: educação on-line, aprendizagem, discurso, multimídia.

\section{INTRODUCCIÓN}

Numerosos estudios se han ocupado de determinar el modo en que el aprendizaje y el lenguaje están relacionados (Flanders, 1970; Sinclair y Coulthard, 1978; Lemke, 1997, Marton et al., 2004, Christie, 2002; Martin y Rose, 2007). Sin embargo, en la actualidad, con los avances de las Tecnologías de la Información y la Comunicación y el continuo desarrollo de dinámicas interactivas multimediales en sitios webs y plataformas de e-learning, dicha relación adquiere una nueva y singular vigencia. El presente estudio, entonces, explora la relación que se establece entre el aprendizaje y el discurso online. En este sentido, se asume la conceptualización de aprendizaje ofrecida por Marton et al. (2004) y, en consonancia con esta conceptualización, se analiza el discurso de acuerdo a los principios fundamentales de las semiótica social multimedial. Desde esta perspectiva, se presta especial atención a las estrategias (Lemke, 1997) discursivas, hipertextuales y multimediales utilizadas por docentes, tutores y alumnos para configurar los llamados espacios de aprendizaje (Marton et al., 2004) en las diferentes materias de un Máster interuniversitario en formación de profesorado de calidad para la docencia preuniversitaria (MIFORCAL).

\section{SOBRE EL ESTUDIO DE LA RELACIÓN ENTRE APRENDIZAJE Y DISCURSO}

El interés en el lenguaje como herramienta para transmitir conocimiento sociocultural y como medio para socializar en formas de pensar y actuar ha incentivado el estudio de los procesos de enseñanza y aprendizaje (Rosemberg, 2000).

Los enfoques tradicionales sobre el modo en el que la interacción verbal incide en el aprendizaje (Flanders, 1970; Sinclair y Coulthard, 1978; Stubbs, 1997) analizan el rol del adulto como informante que suministra conocimiento al estudiante, modela el desempeño apropiado y evalúa los intentos del estudiante por realizar la tarea (Rosemberg, 2000).

En la actualidad se ha dado un remozamiento de algunas perspectivas "tradicionales" y una revitalización a favor del posicionamiento de otras perspectivas para el análisis del discurso didáctico (Christie, 2002; Martin \& Rose, 2007; Marton et al., 2004), con fundadas pretensiones interpretativas y potencialidades prescriptivas que son congruentes con los planteos didácticos actuales, particularmente con aquellos derivados de la corriente cognitiva que otorgan un rol importante a la interacción verbal en el desarrollo del pensamiento (Constantino, 2003). Así las perspectivas actuales, lejos de considerar al aprendizaje como un simple traspaso de conocimiento del adulto al niño, sostienen, por el contrario, que el aprendizaje se mediatiza a través de procesos interactivos -tanto verbales como no verbales- muy complejos (Rosemberg, 2000). A los fines de esta investigación, nos interesan los estudios realizados por Marton et al. (2004) y, en particular, su conceptualización sobre el aprendizaje y el rol que adjudican al lenguaje en dicho proceso.

Marton et al. (2004) se ocupan del aprendizaje en la escuela y de la función del lenguaje en el aprendizaje. Desde su punto de vista, el punto de partida de los acciones 
pedagógicas está representado por las capacidades que se deberían desarrollar. En este sentido, los autores destacan que el aprendizaje siempre significa la adquisición de un conocimiento sobre "algo", que es el objeto del aprendizaje. Este objeto está representado por una capacidad que tiene un aspecto general y otro específico. El aspecto general se refiere a la naturaleza de la capacidad, como recordar, discernir, interpretar, etc. El aspecto específico está determinado por el tema, como problemas de ingeniería o la Segunda Guerra Mundial.

El aprendizaje, sin embargo, no solo es entendido como la adquisición de "algo", sino que, según los autores, también es un proceso por el cual, como resultado de ciertas experiencias, uno se vuelve capaz de manejarse en nuevas situaciones de forma potente. Los modos en que las personas actúan de forma potente están determinados por la manera en que perciben las situaciones y, para percibir las situaciones, se deben discernir diferentes aspectos de las mismas, lo que requiere experimentar variaciones.

Sobre la base de los conceptos anteriores, la tesis fundamental de Marton et al. (2004) es que las diferencias en que los estudiantes aprenden dependen de lo que pueden aprender, y lo que pueden aprender es un espacio de aprendizaje constituido por aquello que puede discernir. Discernir implica reconocer variaciones y para esto es necesario experimentar al mismo tiempo las diferentes instancias en que algo se encuentra en diferentes momentos. Marton et al. (2004) distinguen cuatro patrones de variación:

1. Contraste: una persona debe comparar "algo" con otra cosa a fin de experimentarlo. Por ejemplo, para comprender el "número tres" se lo debe comparar con el cuatro y el cinco.

2. Generalización: Para comprender el "número tres", por ejemplo, se debe comprender este concepto en diferentes situaciones, como tres manzanas, tres autos, tres libros, etc.

3. Separación: para experimentar un aspecto de "algo", es necesario separar este aspecto de otros aspectos. Es decir, este aspecto debe variar mientras otros permanecen estables.

4. Fusión: en ocasiones es necesario que el estudiante experimente diferentes aspectos al mismo tiempo para comprender "algo".

Respecto del concepto de variación, cabe destacar que el lenguaje no sólo permite representar las experiencias, sino también constituirlas y modificarlas. Así un mismo fenómeno se puede expresar de maneras cualitativamente diferentes y estas diferencias se pueden reconocer en los recursos lingüísticos empleados.

A partir de los conceptos expuestos, los autores estudian las preguntas de la docente, la dimensión semántica y el espacio compartido del aprendizaje. En cuanto al espacio compartido del aprendizaje, explican que se trata de un espacio compartido en tanto el objeto de aprendizaje depende de la posibilidad de aprender a partir de lo que está ocurriendo en clase. Este espacio, entonces, puede presentar algunos inconvenientes debido a cuestiones lingüísticas o, también, a que el discurso genera que los participantes focalicen diferentes lugares de la conciencia. Es importante tener en cuenta que, en el aula, si bien lo que se focaliza en la conciencia de los alumnos suele depender del docente, en ocasiones los espacios compartidos de aprendizaje se constituyen en forma colaborativa. A continuación se presenta un ejemplo propuesto por Marton y Tsui (2004:180) en el que participan la docente $(\mathrm{T})$ y un alumno $(\mathrm{S})$ : 


\section{Ejemplo 1}

1. T: Entonces la gente con el mismo apellido permanece junta. ¿Por qué permanece

2. junta? ¿Por qué permanecen juntas? ¿Por qué?

3. S: Conveniencia

4. T: Es conveniente. ¿Podría ser mejor para ellos estar separados? ¿Podría ser mejor

5. para ellos seguir sus propios caminos? ¿Quién dijo que es conveniente? ¿Cuáles son

6. las ventajas de vivir juntos?

7. S: Se pueden ayudar entre ellos

8. T: Ayuda entre ellos. Sí, es verdad. Muy bien... ¿Qué tipo de cosas necesitan para

9. ayudarse mutuamente? Les pregunto a todos

10. S: Cultivar

11. T: Sí, cultivar, ellos se necesitan mutuamente. Vos trabajás en esta parte (del campo)

12. y yo trabajo de la otra. Nos ayudamos mutuamente. ¿Qué más, además del cultivar?

13. S: Cazar

14. T: Cazar. OK. Pero sabemos que era aún una sociedad agrícola, por lo tanto no

15. había mucha caza-

En este caso, las preguntas del docente proponen oportunidades para que los estudiantes exploren los motivos por los cuales un grupo de personas ha permanecido unido en clanes. Los estudiantes aprovechan estas oportunidades y el docente, a su vez, toma sus intervenciones para abrir nuevas dimensiones de exploración.

De esta maneta, a partir de diferentes casos, Marton et al. (2004) muestran de qué manera el lenguaje contribuye a establecer las condiciones necesarias para que los estudiantes aprendan y también a facilitar que ellos tomen conciencia de los aspectos básicos del objeto de aprendizaje. Como se puede observar, estos autores están especialmente interesados en el objeto de aprendizaje que deriva de aquello que la docente y los alumnos dicen y hacen, es decir, del modo en que estructuran las condiciones del aprendizaje. Desde este punto de vista, investigar el aprendizaje es estudiar el modo en que los estudiantes experimentan el objeto de aprendizaje y esto comprende el análisis de las oportunidades de experimentarlo que se les brinda, es decir, el análisis de los espacios de aprendizaje.

Esta investigación parte, entonces, de esta conceptualización del aprendizaje y de los espacios de aprendizaje, destacando que se hablará, por un lado, de "aprendizaje en sentido restringido", cuando se refiere a la comprensión de un conocimiento sobre "algo" (i.e. el objeto de aprendizaje); y, por otro lado, de "aprendizaje en sentido más amplio", cuando se alude a la posibilidad de que los estudiantes actúen en formas potentes a partir de ciertas experiencias.

\section{SOBRE EL ESTUDIO DE LA RELACIÓN ENTRE APRENDIZAJE Y DISCURSO ONLINE}

A partir del aumento de las ofertas educativas en modalidad online, se ha incrementado también la preocupación por los aspectos pedagógicos en ambientes virtuales, así como la investigación sobre este tema. Uno de los focos fundamentales de las investigaciones es el estudio de la interacción comunicativa, lo que apunta a entender cómo se producen las transformaciones en la manera de comunicarnos y de qué forma dichas transformaciones inciden en los procesos pedagógicos (Alvarez Cadavid et al., 2010). 
Algunos de estos trabajos se interesan por clasificar las intervenciones según los procesos de pensamiento (Henri, 1992; King y Kitchener, 1994; Newman et al., 1996; Perry, 1999), teniendo, como base teórica, las teorías cognitivas centradas en las capacidades de procesamiento de información y las estructuras de pensamiento para la construcción de conocimiento y logro de aprendizajes. Otros estudios, en cambio, se ocupan más del trabajo grupal y el proceso discursivo (Constantino, 2002a; Gunawardena et al., 1997; Sánchez-Upegui, 2009) y sus bases teóricas se encuentran en las teorías socioconstructivistas del aprendizaje, que tienen su punto de mira en la interacción social mediada, la cual permite, poco a poco, aumentar los niveles de conocimiento.

Sin duda, las propuestas recién analizadas constituyen una aproximación válida al análisis del discurso didáctico online. Sin embargo, ninguna de ellas ha sido realizada conforme a los criterios de la semiótica social multimedial (Kress, 2004), que puede contribuir con la comprensión no solo de los aspectos puramente verbales, sino también de aquellos relativos a la multimedialidad característica de los contextos online.

De acuerdo con lo expuesto, y partiendo de la conceptualización de aprendizaje propuesta por Marton et al. (2004), este trabajo propone estudiar, desde la semiótica social multimedial (Baldry and Thibault, 2006; Kress, 2004; O'Halloran, 2009), las diversas modalidades de constitución de los espacios de aprendizaje en entornos online.

\section{LA SEMIÓTICA SOCIAL: ENFOQUE PARA EL ANÁLISIS DEL DISCURSO DIDÁCTICO EN AMBIENTES DE APRENDIZAJE EN RED}

Como indican Kress y van Leeuwen (2001: 1), del siglo XX al XXI se establece un cambio desde los textos monomediales a las prácticas semióticas sociales multimediales. Esto explica, al menos en parte, que hayan surgido diversos análisis multimediales: desde textos impresos (e.g. Baldry and Thibault, 2006) hasta sitios de internet (e.g. Baldry and Thibault, 2006; Djonov, 2007; Lemke, 2002). Así se configura un campo llamado "multimedialidad" que supone que la semiosis social se lleva a cabo a partir de múltiples medios.

En este campo se ha recurrido con frecuencia a la teoría sistémico funcional (Halliday, 1982[1978], 1994[1985]) como base para los análisis, lo que ha dado lugar a la semiótica social multimedial, una perspectiva desde la cual se pretende proporcionar esquemas para conceptualizar la selección compleja de recursos semióticos empleados para producir significado (e.g. lenguaje, imágenes, gestos, sonidos, música), así como prácticas para analizar los significados que surgen del uso integrado de esos recursos en artefactos comunicativos (O'Halloran, 2009).

Desde esta perspectiva, los recursos semióticos, que abarcan no sólo lo lingüístico sino también imágenes, símbolos, música, sonido, espacio, etc., son elementos usados para producir significado. Un sistema de recursos semiótico, por otra parte, comprende un sistema de posibles significados y formas típicamente empleadas para producir significado en un determinado contexto (Baldry y Thibault, 2006:18).

En este marco, resulta fundamental el concepto de estrategia (o táctica) dado que, cuando realizamos cualquier tipo de acción social significativa, utilizamos recursos estratégicamente [...] dentro de ciertos límites tenemos una amplia libertad para producir una gran variedad de diferencias sutiles en los significados, a través de lo que hacemos y el modo en que lo hacemos (Lemke, 1997: 208). 
Así, en el presente trabajo, desde la semiótica social multimedial, se pretende mostrar de qué manera recursos semióticos de diferente naturaleza son utilizados estratégicamente para crear espacios de aprendizaje en entornos online.

\section{METODOLOGÍA}

En consonancia con las cuestiones planteadas y el objetivo propuesto, la metodología es de carácter netamente cualitativo (Constantino, 2002b; Guba y Lincoln, 1998; Vasilachis de Gialdino, 2007 [2006]) y encuadrable en los principios -recién mencionados- de Marton et al. (2004) y de la semiótica social multimedial.

El corpus principal de la investigación proviene del Máster interuniversitario en formación de profesorado de calidad para la docencia preuniversitaria (MIFORCAL). ${ }^{1}$ La estructura curricular adoptada para el proyecto corresponde a una concepción epistémica y de organización didáctica que distingue y articula tres niveles de conocimientos diferentes y complementarios: Teoría, Didáctica y Laboratorio (identificados como T, D y L, respectivamente). Por T, se entienden los fundamentos históricos-epistemológicos de la disciplina o área disciplinar propuesta; la $\mathrm{D}$ es concebida sintéticamente como la teoría de la praxis o acción docente, también contextualizada por la disciplina o área disciplinar propuesta más otros factores propios de la situación de enseñanza; el L es conceptualizado como los conocimientos aplicados a circunstancias determinadas en contextos concretos (diseños, planes o proyecciones, simulaciones, estudios de caso, etc.). Esta tríada (T-D-L) se repite, con diverso peso específico, en todas las secciones temáticas del Área Común y de los Ciclos de Formación Orientados.

Para este trabajo nos centraremos en una de las orientaciones del Máster: Ciencias Sociales y Humanas. Esta orientación cuenta con nueve materias: una materia de Didáctica de las Ciencias Humanas y otra de las Ciencias Sociales y, en relación con cada una, una materia correspondiente al Laboratorio y otra a los Fundamentos. Además, hay una materia sobre Metodología de la Investigación que tiene, a su vez, un Laboratorio y una materia de Fundamentos.

El material de cada materia está a cargo de un/a docente que, solo en algunos casos, participa junto con la persona a cargo de la tutoría de los foros. Por otra parte, la tutoría de los foros de cada materia está a cargo siempre de la misma persona.

Esta investigación se lleva a cabo por fases, durante las cuales se aplican diferentes métodos y técnicas para el relevamiento y el análisis de los datos:

- Recolección y sistematización del corpus: descripciones del diseño de los cursos y relevamiento de los materiales, los foros y las chats;

- Observación y lectura analíticas y sucesivas de los cursos, sus materiales, foros y chats, desde primeras impresiones - sin un trabajo de encuadre teórico sino más bien de captación de la dinámica global y los significados manifiestos- hasta relecturas

Este master es gestionado por una red institucional latinoamericana y europea a partir de un proyecto ALFA de la UE, con sede administrativa en la Universidad Ca' Foscari di Venecia, y sede operativa en Argentina en el Centro de Investigaciones en Antropología Filosófica y Cultural (CIAFIC), dependiente del Consejo Nacional de Investigaciones Científicas y Técnicas (CONICET). 
con diferentes criterios e instrumentos conceptuales derivados de la semiótica social multimedial;

- Análisis de los cursos teniendo en cuenta los conceptos mencionados;

- Clasificación y caracterización de estrategias discursivas, hipertextuales y/o multimediales orientadas al establecimiento de espacios de aprendizaje.

Respecto de los ejemplos citados, cabe destacar que se han modificado los nombres para preservar la identidad, y que se han respetado los mensajes originales.

\section{ESTRATEGIAS DISCURSIVAS, HIPERTEXTUALES Y/O MULTIMEDIALES ORIENTADAS A LA CREACIÓN DE ESPACIOS DE APRENDIZAJE}

Retomando lo expuesto previamente, crear un espacio de aprendizaje es abrir una dimensión de variación (respecto de lo tomado como natural en ausencia de esa variación). El espacio de aprendizaje, por otra parte, nos indica lo que es posible aprender en una situación determinada. Veremos a continuación las diferentes estrategias de los tutores, en algunos casos también los docentes, y los estudiantes para generar espacios de aprendizajes.

\subsection{ESTRATEGIAS EMPLEADAS DENTRO O FUERA DEL FORO POR EL RESPONSABLE DE LA TUTORÍA}

Es fundamental considerar que el responsable de la tutoría, en algunos casos el mismo docente, propone, ya sea dentro o fuera del foro, realizar una actividad, que genera condiciones para el aprendizaje.

En los casos en que esta propuesta es realizada dentro del foro, el tutor participa con una intervención extensa en la que detalla los puntos fundamentales de la actividad, incluida la fecha de entrega. En estos casos, la materia no suele presentar, en su diseño general, divisiones semanales ni tampoco abarcar una variedad amplia de recursos didácticos, por lo cual todas las acciones didácticas que se deben cumplir aparecen presentadas en los foros.

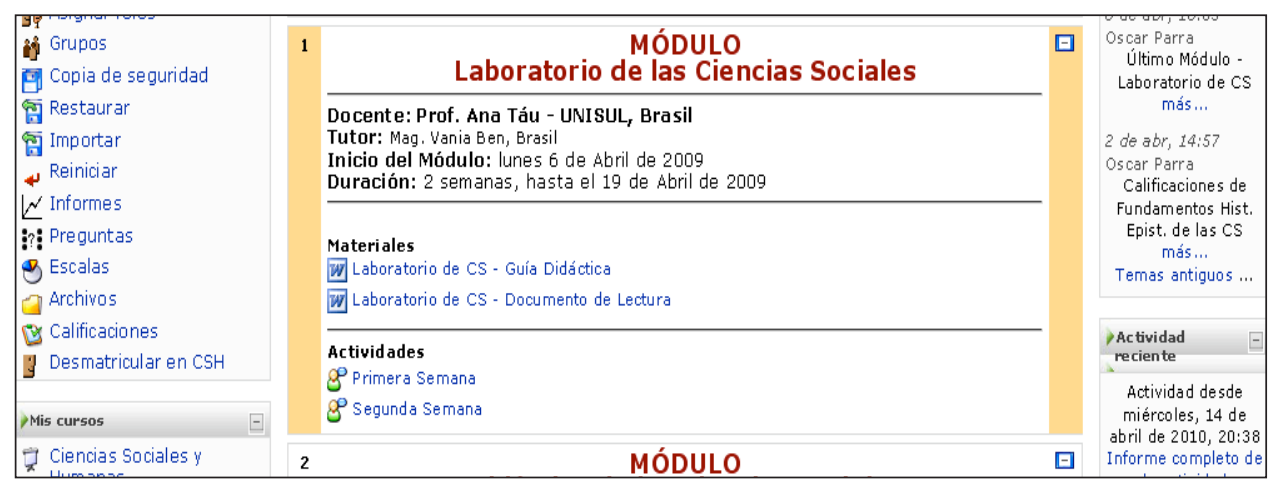

Figura 1. Ejemplo de materia sin divisiones semanales ni presencia de variedad de recursos didácticos. 
En la Figura 1 se muestra una materia sin divisiones internas y, a continuación, la intervención con la cual la docente presenta la actividad.

En la Figura 1, entonces, se presenta la materia Laboratorio de las Ciencias Sociales, que no incluye divisiones semanales. En este sentido, el tutor de los foros propone las actividades en algunas de sus intervenciones en el foro, como en el siguiente ejemplo:

\section{Ejemplo 2}

Mapa Conceptual de Vania Ben - lunes, 13 de abril de 2009, 02:38

¡Constantes Animadores!

Les propongo la construcción de un Mapa Conceptual, presentando un esquema investigativo de su trabajo de tesis. Bien, como los elementos claves que constituyen el estudio. El objetivo es unir las aportaciones del material de la Profesora Tau a los pasos de la tesis. Inicialmente les invito a que hagan dos borradores del mapa, y discutan con los compañeros en el foro, enseguida el tercero como tarea para entregar, debe ser puesto en archivo en el foro así: su nombre.

Mapa Conceptual 3.

Quiero aclarar que este mapa debe ser bien hecho, pues para que el mismo sirva como esquema mental, y en la presentación y defensa de la tesis...

Estamos en la recta final de este módulo, más una semanita y ya estaremos concluyendo. $Y$ a seguir con la aventura, con mucha garra, constancia y sabiduría en la tesis, que seguro cada uno expresará sus visiones y pensamientos para la Sociedad del Conocimiento, en nuestra gran familia MIFORCAL.

¡Ánimo y adelante!

Abrazo afectuoso, Vania

En otros casos, en cambio, cuando las materias sí tienen divisiones por semana e incluyen diversos recursos didácticos, el tutor anticipa algunos puntos de la tarea en la etiqueta del módulo semanal y, además, utiliza el recurso didáctico "Actividad" que ofrece la plataforma. Esto se puede observar en la materia Laboratorio de Metodología de la Investigación en Ciencias Sociales.

En la Figura 2 se presenta el diseño del Laboratorio de Investigación en Ciencias Sociales, una materia con divisiones por semanas, que emplea diversos recursos didácticos.

En este sentido, es posible observar, que la actividad se anticipa y presenta en espacios diferentes al foro. Dicha anticipación se da ya en la etiqueta asociada a cada semana:

\section{Ejemplo 3}

Durante la segunda semana (del 11 al 18 de septiembre), nuestro trabajo consistirá en analizar diferentes opciones teóricas y metodológicas en el proceso de una investigación concreta. Las actividades para el logro de este objetivo se realizarán en el siguiente orden:

$1^{\circ}$ ) Elaboración de la tarea de aplicación 2 (para la cual deberán adoptar el rol de un investigador que toma decisiones metodológicas).

$2^{\circ}$ ) Una vez entregada la tarea de aplicación, lectura y análisis de la segunda parte del módulo. Para acompañar esta tarea se abrirá un foro de debate.

Por otro lado, se recurre al recurso "Actividad" para presentar las pautas de dicha actividad: 


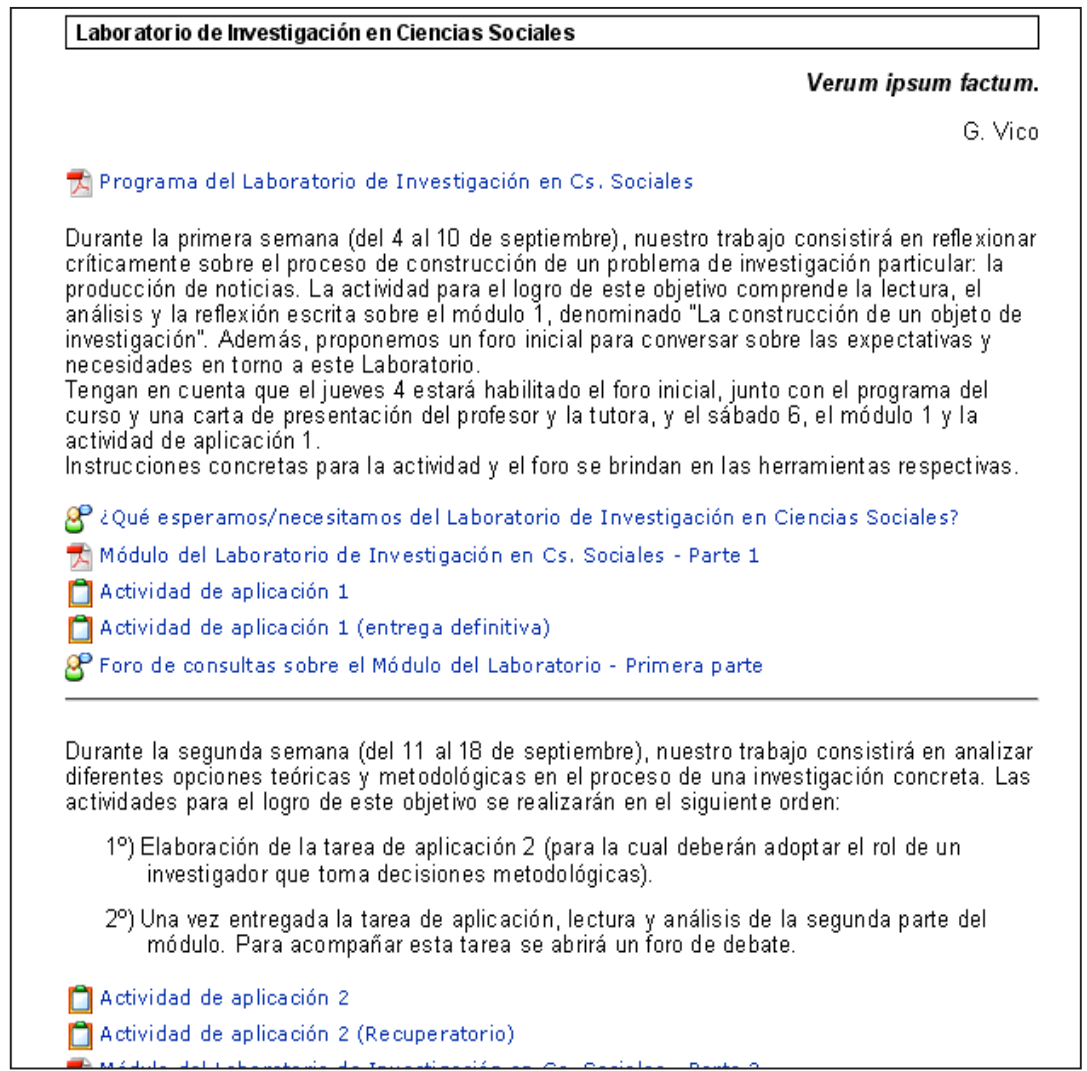

Figura 2. Ejemplo de materia con divisiones semanales y uso de múltiples recursos didácticos.

\section{Ejemplo 4}

Para realizar esta tarea deben imaginar que son los investigadores responsables de la investigación presentada en la primera parte del módulo (es decir, la investigación realizada por Dario Soria).

Les pedimos, entonces, que, como si fueran los investigadores, escriban un texto breve (aproximadamente 500 palabras) que describa la manera en que desarrollarían la investigación: cómo plantearían la relación sujeto-objeto o sujeto-sujeto, qué técnicas de recolección de datos emplearían, etc.

\section{IMPORTANTE:}

- Por favor, al subir la tarea, nombren el archivo de la siguiente manera: Apellido actividad 2. Por ejemplo, Mora Actividad 2.

- Por favor, suban esta tarea, a más tardar, martes 16 a la mañana. Así podemos continuar con el desarrollo de esta unidad. 
Además de estas diferencias relativas a los aspectos hipertextuales y multimediales, también es relevante destacar, desde el punto de vista estratégico, las diversos tipos de actividades que proponen los responsables de la tutoría:

- Elaboración de resumen o síntesis del material leído.

- Elaboración de un mapa conceptual teniendo en cuenta el material leído y analizado. En estos casos, el tutor solicita que los alumnos muestren sus mapas en el foro $\mathrm{y}$, desde allí, expone posibles correcciones.

- Reconocimiento de conceptos puntuales, en algunos casos fundamentales, de los materiales leídos y explicación de los mismos. En algunos casos, esta discusión de conceptos aparece guiada por el trabajo inicial con un concepto particular.

- Elaboración de un glosario que incluya conceptos que se han discutido en los foros.

- Diseño de una programación didáctica y reflexión sobre esta programación. En este caso, el diseño se propone en Didáctica y la reflexión en el Laboratorio de Didáctica.

- Propuesta de ponerse en el lugar de un investigador y reflexionar, así, en torno los conceptos leídos sobre metodología. Esto surge en un Laboratorio.

Además de plantear diferentes tipos de actividades que deben ser realizadas fuera del foro, el docente o el tutor emplean diferentes estrategias durante la interacción comunicativa del foro.

\subsection{ESTRATEGIAS EMPLEADAS DENTRO DEL FORO POR LOS TUTORES O LOS ESTUDIANTES}

6.2.1. ESTRATEGIAS DEL RESPONSABLE DE LA TUTORÍA

Las estrategias empleadas dentro del foro por el encargado de la tutoría apuntan a lo siguiente:

- Formular preguntas que apunten a la reflexión sobre el material leído y faciliten su comprensión.

- Generar la discusión de los conceptos extraídos de la lectura propuesta. Incluso, en algunos casos, esta discusión apunta a la creación de un glosario.

\section{Ejemplo 5}

\section{Bienvenida}

de Marta Gauna - lunes, 16 de marzo de 2009, 15:29

Bienvenidos al Módulo:

Queridos compañeros de viaje:

Hacia el final de la jornada de estudios, pero no de aprendizaje, y antes de la aplicación conceptual en una tesis, estaré con ustedes estas semanas.

La primera tarea es leer y discutir sobre los conceptos generales.

Les estoy esperando.

MARTA 
- Formular preguntas a partir de tópicos o cuestiones que los alumnos plantean durante el desarrollo de la interacción.

\section{Ejemplo 6}

Re: Foro de consultas sobre el Módulo del Laboratorio - Primera parte

de Sebastián Sayago - miércoles, 10 de septiembre de 2008, 00:48

Hola, Manuela.

Has realizado una muy buena primera interpretación del módulo.

En este juego de preguntas y respuestas, de idas y vueltas entre la lectura y la escritura, planteo la siguiente inquietud: ¿estás segura de que la concepción de realidad que subyace a la definición del objeto de estudio es relativista? ¿En qué sentido?

Saludos.

Sebastián

Además de las estrategias relevadas, aparece otro tipo de estrategia luego de que los alumnos manifiestan haber tenido inconvenientes en el proceso de aprendizaje. En el caso de los tutores, estas estrategias apuntan a:

- Responder las preguntas planteadas por los alumnos.

- Solicitar la reescritura de los trabajos.

- Ante dudas en las que se transfiere lo propuesto teóricamente a la propia realidad, apelar a que el trabajo en el módulo permitirá dar respuesta.

- Proponer a los alumnos que revisen la actividad con errores y reelaboren a partir de las observaciones.

\subsubsection{ESTRATEGIAS EMPLEADAS POR LOS ESTUDIANTES}

En cuanto a los alumnos, durante la interacción comunicativa del foro, emplean las siguientes estrategias:

- Formular preguntas para verificar dudas acerca de aspectos de las tareas que no les hayan quedado claros. El Ejemplo 7 ilustra este caso:

\section{Ejemplo 7}

Re: La didáctica

de Rodolfo Pa - viernes, 20 de marzo de 2009, 15:11

Disculpe profesora, podría aclarar más lo del resumen que está solicitando. Tenemos que resumir todo el texto? tenemos que resumir los conceptos básicos?

Le pregunto porque la primera indicación fue discutir los conceptos básicos.

Hasta cuando tenemos tiempo para los resúmenes?

Gracias.

En general, se plantea inicialmente cuál es la confusión y luego se solicita aclaración sobre dicho aspecto por medio de preguntas.

- Plantear preguntas sobre un concepto puntual.

- Explicar un concepto como forma de verificar, a partir de esta explicación, si se ha comprendido o no lo leído. En ocasiones, el pedido de verificación es más explícito 
y se realiza por medio de preguntas. En otros casos, este pedido de verificación no es explícito, sino que está sugerido por medio de frases o giros, como en el Ejemplo 8.

\section{Ejemplo 8}

Didáctica de las Ciencias Sociales

de Rodolfo Pa - miércoles, 18 de marzo de 2009, 13:24

Me parece sumamente rico los esquemas presentados en el material (pp. 29-35) sobre las investigaciones de Shulman, quien afirma que la formación docente no es capacitar para actuar de la manera prescrita, sino capacitar para razonar correctamente. Esto complejiza la tarea de los institutos de formación docente y viendo los últimos resultados en las evaluaciones del MEC, sería ideal reflexionar sobre los conocimientos que el profesor debe adaptar a las situaciones del PEA.

Comprensión-Transformación-Enseñanza-Evaluación-Reflexión-Comprensión

La crítica al didácticismo me parece muy acertada y me parece necesario rescatar el contenido como elemento importante en la enseñanza. Por lo que estoy leyendo, es el contenido quien va a fundar, epistemológicamente hablando, la didáctica especial.

En este caso, el alumno hace referencia a una serie de ideas que extrae del material de lectura y emplea frases, particularmente "por lo que estoy leyendo", que sugieren que está verificando con los lectores del foro si su comprensión es adecuada.

- Plantear dudas acerca de lo leído en el material de la materia o en el mismo módulo a partir del intento de transferir los conceptos a la propia realidad.

\section{Ejemplo 9}

Re: Ampliación del plazo de participación en el Foro de Mercerdes Aquino - martes, 23 de septiembre de 2008, 06:10

Hola Gastón, Griselda y compañeros: muy interesante la lectura del aspecto metodológico; la lentitud del análisis no está en el material que es bastante claro y ejemplificador, sino la reflexión concomitante que suscita; pues uno se ubica en el contexto de investigador y es donde surge la preocupación del verdadero alcance de hacer una investigación desde esta perspectiva. Se requiere claridad conceptual y metodológica; y por sobre todo reciedumbre, organización y voluntad.

Acercarse al objeto de estudio en diferentes niveles, desde diferentes sustentos teóricos y utilizando formas variadas de recolección exige real competencia del investigador. Creo que los conocimientos teóricos son fundamentales en todos los niveles de análisis. Mi cuestionamiento - preocupación más que pregunta, se viene en cuanto a cómo integrar diferentes datos capturados para el corpus de análisis.

Me ubico en el tema que quiero investigar en la facultad -el uso de estrategias cognitivas e de aprendizaje por estudiantes del primer año de la universidad. ¿Còmo recoger un corpus de base interesante?

- En los casos en los alumnos están trabajando solos en un foro, suelen formular preguntas u orientar las actividades como lo haría la docente.

Ejemplos 10 


\section{Trabajando con filósofos}

di Moria Lopez - Tuesday, 20 October 2009, 21:33

Hola compañeros, para encauzar el trabajo, que por cierto es bastante arduo, les propongo que primero alcemos una lista de los posibles filósofos, para luego elegir al más popular con quien trabajaremos en última instancia, sólo que la elección a mi parecer no tendría que pasar de mañana por que el tiempo aprecia, espero noticias.

Va mis primeros candidatos: Descartes, Leibniz, Spinoza y Kant

Además de las estrategias mencionadas, y al igual que en el caso de los responsables de la tutoría, los estudiantes despliegan algunas estrategias particulares una vez que alguno de ellos ha manifestado inconvenientes en el proceso de aprendizaje. Así:

- Cuando un alumno realiza preguntas al docente, algunos de los compañeros suelen responder en su lugar. Luego, generalmente, el docente corrobora lo dicho por el alumno.

- Cuando los alumnos están trabajando solos y un alumno dice no haber entendido la pregunta de otro, éste reformula su pregunta.

\subsection{OBSERVACIONES GENERALES}

De acuerdo con el análisis realizado, en relación con las estrategias que apuntan a la creación de espacios de aprendizaje, tanto en el caso de los tutores como de los alumnos, es notorio el poco uso de recursos multimediales e hipertextuales. En general, los participantes tienden a la realización de intervenciones verbales en los foros. En raras ocasiones recurren a la multimedialidad y la hipertextualidad. En este caso, solo se relevaron recursos multimediales en dos casos. Por un lado, cuando los tutores anticipan las actividades en las etiquetas del módulo y las presentan en un espacio específico. Por otro lado, cuando los alumnos, a pedido del tutor, elaboran un mapa conceptual y lo suben al foro. Dicha escasez de recursos y estrategias multimediales llama la atención teniendo en cuenta que una de las ventajas del medio electrónico es la posibilidad de usar recursos de diferente naturaleza.

Por otra parte, se ha verificado que las estrategias de los responsables de la tutoría apuntan, fundamentalmente a un tipo de "aprendizaje restringido", es decir, buscan asegurar y/o verificar la comprensión del objeto de aprendizaje, sea en su aspecto específico sea en su aspecto general. Así, se suelen desplegar estrategias para corroborar si los alumnos han entendido las ideas y conceptos propuestos en el material de lectura. El patrón de variación establecido en este tipo de tareas es el que corresponde, sobre todo, a la fusión de aspectos, en tanto se pretende que los alumnos comprendan, por ejemplo, el conjunto de aspectos característicos de un concepto.

En menor medida, los docentes emplean estrategias para asegurar el "aprendizaje en un sentido amplio", es decir, la posibilidad de que los alumnos, en base a los conceptos aprendidos, puedan actuar en formas potentes en nuevas situaciones. En este sentido, se observa que solo en dos casos se proponen actividades en las cuales los estudiantes deben intentar aplicar las ideas vistas en los materiales en nuevas situaciones, como cuando se les solicita que elaboren una programación didáctica o que se pongan en el lugar de un investigador y diseñen la metodología para una investigación. En ambos casos, el patrón de variación es el de la generalización, en la medida que se pretende que un concepto 
aprendido en relación con determinado contexto se aplique en otros. Por otra parte, cabe destacar que este tipo de estrategia es más frecuente en las materias de Laboratorio.

En cuanto a las estrategias de los alumnos, es importante destacar que no se verifica el uso de estrategias hipertextuales y multimediales. Además, se ha observado que las estrategias, que son exclusivamente verbales, tienden a centrarse más en el "aprendizaje en un sentido más restringido". En este sentido, apuntan a develar dudas puntuales sobre la consigna o sobre alguna idea o algún concepto del material leído. En este caso, se pone de manifiesto la fusión como patrón de variación característica del espacio de aprendizaje. Sin embargo, aun cuando ese tipo de estrategia es muy frecuente, en ocasiones los alumnos intentan relacionar los conceptos leídos con su experiencia cotidiana y personal, apuntando así a un "aprendizaje más amplio". En estos casos, el patrón de variación suele ser el de la generalización.

Respecto a las intervenciones de los alumnos, cabe destacar también que la frecuencia en que emplean estrategias para manifestar sus dudas o confusiones es más alta cuando los materiales o el foro apuntan a comprender ámbitos desconocidos para ellos.

Por último, al comparar las estrategias de los alumnos con las de los tutores, observamos que, si bien son diferentes, apuntan en general a un tipo de "aprendizaje restringido" y suponen implícitamente patrones de variaciones similares: en mayor medida, la fusión y, en menor medida, la generalización.

\section{CONCLUSIÓN}

En el presente artículo se ha analizado la relación entre el aprendizaje y el discurso online, considerando la conceptualización de aprendizaje ofrecida por Marton et al. (2004) y los principios fundamentales de las semiótica social multimedial. Desde esta perspectiva, hemos enfocado las estrategias (Lemke, 1997) discursivas, hipertextuales y multimediales utilizadas por docentes, tutores y alumnos para configurar los llamados espacios de aprendizaje (Marton et al., 2004) en las diferentes materias de un Máster interuniversitario en formación de profesorado de calidad para la docencia preuniversitaria (MIFORCAL).

Este análisis muestra que las estrategias hipertextuales y multimediales son infrecuente y solo empleadas por los tutores (que en algunos casos coinciden con los docentes). Por otra parte, tanto en el caso de los tutores como en el de los alumnos, las estrategias apuntan, fundamentalmente, al "aprendizaje en un sentido restringido". En estos casos, el patrón de variación característico de los espacios de aprendizaje es la fusión. También notamos que, solo en algunas ocasiones, las estrategias apuntan a un aprendizaje más amplio, es decir, a que los estudiantes puedan a aplicar los conceptos de manera potente en nuevas situaciones.

De acuerdo con lo analizado, es posible sugerir que en entornos de aprendizaje online deberían utilizarse más cantidad de estrategias que combinen recursos verbales con otro tipo de recursos (hipertextuales y multimediales). También sería conveniente que estas estrategias apuntaran, con más frecuencia, a un "aprendizaje en sentido amplio".

Finalmente, este análisis permitió una primera consideración de las estrategias utilizadas para crear espacios de aprendizaje. Será interesante, luego de este trabajo, observar y analizar estas estrategias en un corpus más amplio. Así, en la próxima etapa de la investigación extenderemos el análisis a nuevos entornos virtuales. 


\section{REFERENCIAS BIBLIOGRÁFICAS}

Álvarez Cadavid, G.; Alvarez, G.; Constantino, G.D.; Raffaghelli, J. Concepciones, modelos y propuestas alternativas para la investigación del aprendizaje en red: Una aproximación desde la experiencia del proyecto REDES. En Constantino, G.D, Cibercultura : formación e investigación en la web. Buenos Aires : Editorial Universitaria Rioplatense.

Baldry, A. Thibault, P.J. (2006). Multimodal Transcription and Text Analysis. Londres: Equinox.

Christie, F. (2002). Classroom Discourse Analysis. Nueva York: Continuum.

Constantino, G. (2002a). Presenza vitale contro presenza virtuale: studio contrastivo dei modi strategico-discorsivi della interazione didattica. En M. Banzato (ed.), Apprendere in rete. Torino: UTET.

Constantino, G.D. (comp.) (2002b). Investigación Cualitativa \& Análisis del Discurso en Educación. Catamarca: Universitaria.

Flanders, N. (1977). Análisis de la interacción didáctica. Salamanca: Anaya.

Gunawardena, C., Lowe, C., Y Anderson, T. (1997). Analysis on global online debate and the development of an interaction analysis model for examining social construction of knowledge in computer conferencing. Journal of Educational Computing Research, vol. 17, n. 4: 397-431.

Halliday, M.A.K. (1982). El lenguaje como semiótica social. México: Fondo de Cultura Económica.

Halliday, M.A.K. (1994). An Introduction to Functional Grammar. Londres: Edward Arnold.

Henri, F. (1992). Computer conferencing and content analysis. En Kayen, A., Collaborative Learning Through Computer Conferencing. Berlin: Sringer - Verlag.

Guba. E.G. Y Lincoln, Y.S. (1998). Competing Paradigms in Qualitative research. En N.K. Denzin y Lincoln Y.S. The Landscape of Qualitative Research (pp. 195-220). Thousand Oak, CA: Sage.

King, P. M., Y Kitchener, K. (1994). Developing Reflective Judgment. San Francisco: JosseyBass. Consultado el 10 de mayo de 2010 en http://www.amazon.com/gp/reader/1555426298/ ref=sib_dp_pt/104-5182237-5697521\#reader-link

Kress, G. (2004). Reading Images: Multimodality, Representation and New Media. Consultado el 10 de mayo de 2010 en http://www.knowledgepresentation.org/BuildingTheFuture/Kress2/ Kress2.html

Kress, G. Y Van Leeuwen, T. (2001). Multimodal Discourse. Arnold: London.

Lemke, J.L. (1997). Aprender a hablar ciencia. Barcelona: Paidós.

Martin, J.R. Y Rose, D. (2007) Interacting with Text: the role of dialogue in learning to read and write [en línea]. http://www.readingtolearn.com.au/pdf/Interacting\%20with\%20Text.pdf

Marton , F., Tsui, A.B.M. et al. (2004). Classroom discourse and the space of learning. New Jersey: Lawrence Erlbaum.

Newman, D., Webb, B., Y Cochrane, C. (1996). A content analysis method to measure critical thinking in face-to-face and computer supported group learning. Recuperado a partir de _http:// www.qub.ac.uk/mgt/papers/methods/contpap.html_

O'halloran, K. L. (2009). Multimodal Analysis and Digital Technology. En A. Baldry and Montagna (eds.), Interdisciplinary Perspectives on Multimodality: Theory and Practice. Campobasso: Palladino.

Perry, W. (1999). Forms of ethical and intellectual development in the college years. San Francisco: Jossey-Bass. Consultado el 9 de mayo de 2010 en _http://www.amazon.com/gp/ reader/0030813263/ref=sib_dp_pt/104-5182237-5697521\#reader-link

Sinclair, J. Mch. Y Coulthard, R.M. (1978). The system of analysis. En Sinclair, J. Mch y Coulthard, R.M., Towards an Analysis of Discourse. The English used by teachers and pupils, (pp. 19-53). Londres: Oxford University Press.

Sttubs, M. (1987). Análisis del discurso. Análisis sociolingüístico del lenguaje natural. Madrid: Alianza. Vasilachis De Gialdino, I. (coord.) (2007). Estrategias de una investigación cualitativa. Buenos Aires: Editorial Gedisa. 
